\title{
The combination of optical coherence tomography and Raman spectroscopy for tissue characterization
}

\author{
Kokila D. Egodage ${ }^{1}$, Sebastian Dochow ${ }^{1,2}$, Thomas W. Bocklitz ${ }^{1}$, Olga Chernavskaia ${ }^{1}$, \\ Christian Matthäus ${ }^{1,2}$, Michael Schmitt ${ }^{1,2}$, Juergen Popp ${ }^{1,2^{*}}$ \\ ${ }^{1}$ Institute of Physical Chemistry and Abbe Center of Photonics, Friedrich-Schiller University Jena, Helmholtzweg 4, \\ 07743 Jena, Germany \\ ${ }^{2}$ Leibniz Institute of Photonic Technology Jena e.V., Albert-Einstein-Str. 9, 07745 Jena, Germany \\ *e-mail: juergen.popp@ipht-jena.de
}

\begin{abstract}
The visualization as well as characterization of diseased tissue are of vital diagnostic interest for an early diagnosis to increase patients' survival rate. In this study we introduce an imaging device combining optical coherence tomography (OCT) and Raman spectroscopy (RS), allowing to record 2D and 3D OCT cross sectional images of bulk tissue samples, as well as the acquisition of Raman spectra from small areas of interest in order to aid the detection process with molecular information. The design of the OCT/RS imaging device consists of commercially available cage components. The probe head involves a CCD camera chip for visualization purposes and galvanic mirrors for scanning the sample in $\mathrm{x}$ and $\mathrm{y}$ directions with a scan line rate up to $76 \mathrm{kHz}$. The OCT/RS imaging approach has been successfully evaluated by investigating pork samples. OCT and Raman data were correlated and different tissue types within the samples were successfully identified and clustered separately. Finally, pork skin samples with visual defects were characterized. Overall, the presented OCT/RS device allows for recording of large morphological overview OCT images to define points of interest, which are afterwards characterized in more detail on a molecular level by means of Raman spectroscopy. (C) 2015 Samara State Aerospace University (SSAU).
\end{abstract}

Keywords: optical coherence tomography, Raman spectroscopy, probe, multimodal imaging.

Paper \#2503 received 2015.06.20; revised manuscript received 2015.06.29; accepted for publication 2015.06.30; published online 2015.06.30.

\section{References}

1. A. Alwan, "Global status report on noncommunicable diseases 2010," Italy: World health organization (2011).

2. S. Edge, D. R. Byrd, C. C. Compton, A. G. Fritz, F. L. Greene, and A. Trotti, "Cancer Staging Manual," AJCC 7 (2010).

3. M. Kerkhof, H. Van Dekken, E. W. Steyerberg, G. A. Meijer, A. H. Mulder, A. De Bruïne, et al., "Grading of dysplasia in Barrett's oesophagus: substantial interobserver variation between general and gastrointestinal pathologists," Histopathology 50, 920-927 (2007).

4. L. Y. Robles, S. Singh, and P. M. Fisichella, "Emerging enhanced imaging technologies of the esophagus: spectroscopy, confocal laser endomicroscopy, and optical coherence tomography," Journal of Surgical Research 195, 502-514 (2015).

5. O. Old, M. L. Almond, R. Griggs, G. R. Lloyd, J. Horsnell, C. Kallaway, L. Fullwood, K. Das, J. Hutchings, M. Isabelle, C. Kendall, N. Stone, and Hugh Barr, "In Vivo Optical Imaging of Early Cancer and Tumours," in In Vivo Optical Imaging. Vol. 1, W. Liu (Ed.), OMICS Group, California (2014).

6. E. de Boer, N. J. Harlaar, A. Taruttis, W. B. Nagengast, E. L. Rosenthal, V. Ntziachristos, et al., "Optical innovations in surgery," British Journal of Surgery 102, e56-e72 (2015).

7. M. Mogensen, T. M. Joergensen, B. M. NÜRnberg, H. A. Morsy, J. B. Thomsen, L. Thrane, et al., "Assessment of Optical Coherence Tomography Imaging in the Diagnosis of Non-Melanoma Skin Cancer and 
Benign Lesions Versus Normal Skin: Observer-Blinded Evaluation by Dermatologists and Pathologists," Dermatologic Surgery 35, 965-972 (2009).

8. V. R. Korde, G. T. Bonnema, W. Xu, C. Krishnamurthy, J. Ranger-Moore, K. Saboda, et al., "Using optical coherence tomography to evaluate skin sun damage and precancer," Lasers in Surgery and Medicine 39, 687695 (2007).

9. T. Gambichler, A. Orlikov, R. Vasa, G. Moussa, K. Hoffmann, M. Stücker, et al., "In vivo optical coherence tomography of basal cell carcinoma," Journal of Dermatological Science 45, 167-173 (2007).

10. J. M. Olmedo, K. E. Warschaw, J. M. Schmitt, and D. L. Swanson, "Optical coherence tomography for the characterization of basal cell carcinoma in vivo: A pilot study," Journal of the American Academy of Dermatology 55, 408-412 (2006).

11. N. D. Gladkova, G. A. Petrova, N. K. Nikulin, S. G. Radenska-Lopovok, L. B. Snopova, Y. P. Chumakov, et al., "In vivo optical coherence tomography imaging of human skin: norm and pathology," Skin Research and Technology 6, 6-16 (2000).

12. L. Themstrup, C. A. Banzhaf, M. Mogensen, and G. B. E. Jemec, "Optical coherence tomography imaging of non-melanoma skin cancer undergoing photodynamic therapy reveals subclinical residual lesions," Photodiagnosis and Photodynamic Therapy 11, 7-12 (2014).

13. K. Jina, B. William, R. M. Jason, L. Howard, and W. Adam, "Functional optical coherence tomography: principles and progress," Physics in Medicine and Biology 60, R211 (2015).

14. H. Ra, E. González-González, M. J. Uddin, B. L. King, A. Lee, I. Ali-Khan, et al., "Detection of NonMelanoma Skin Cancer by in vivo Fluorescence Imaging with Fluorocoxib A," Neoplasia 17, 201-207 (2015).

15. J. B. Ross, D. Huh, L. B. Noble, and S. F. Tavazoie, "Identification of molecular determinants of primary and metastatic tumour re-initiation in breast cancer," Nat Cell Biol 17, 651-664 (2015).

16. S. Dochow, D. Ma, I. Latka, T. Bocklitz, B. Hartl, J. Bec, et al., "Combined fiber probe for fluorescence lifetime and Raman spectroscopy," Analytical and Bioanalytical Chemistry, doi://10.1007/s00216-015-8800-5 (2015).

17. C. Krafft, S. Dochow, I. Latka, B. Dietzek, and J. Popp, "Diagnosis and screening of cancer tissues by fiberoptic probe Raman spectroscopy," Biomedical Spectroscopy and Imaging 1, 39-55 (2012).

18. E. Drakaki, T. Vergou, C. Dessinioti, A. J. Stratigos, C. Salavastru, and C. Antoniou, "Spectroscopic methods for the photodiagnosis of nonmelanoma skin cancer," Journal of biomedical optics 18, 061221 (2013).

19. K. Eberhardt, C. Stiebing, C. Matthäus, M. Schmitt, and J. Popp, "Advantages and limitations of Raman spectroscopy for molecular diagnostics: an update," Expert Review of Molecular Diagnostics 15, 773-787 (2015).

20. C. Krafft, B. Dietzek, M. Schmitt, and J. Popp, "Raman and coherent anti-Stokes Raman scattering microspectroscopy for biomedical applications," Journal of Biomedical Optics 17, 0408011 (2012).

21. P. C. Ashok, B. B. Praveen, N. Bellini, A. Riches, K. Dholakia, and C. S. Herrington, "Combined information from Raman spectroscopy and optical coherence tomography for enhanced diagnostic accuracy in tissue discrimination," in Biomedical Vibrational Spectroscopy VI: Advances in Research and Industry, 89390L (2014).

22. K. Kong, C. J. Rowlands, S. Varma, W. Perkins, I. H. Leach, A. A. Koloydenko, et al., "Diagnosis of tumors during tissue-conserving surgery with integrated autofluorescence and Raman scattering microscopy," Proceedings of the National Academy of Sciences 110, 15189-15194, (2013).

23. M. Calin, S. V. Parasca, R. Savastru, M. Calin, and S. Dontu, "Optical techniques for the noninvasive diagnosis of skin cancer," Journal of Cancer Research and Clinical Oncology 139, 1083-1104 (2013).

24. C. Krafft, B. Dietzek, and J. Popp, "Raman and CARSmicrospectroscopy of cells and tissues," Analyst 134, 1046-1057 (2009).

25. J. Popp, M. Schmitt, B. Dietzek, C. Krafft, R. Möller, and P. Rösch, "Raman meets medicine: Raman spectroscopy: a powerful tool in biophotonics,", 75030N (2009).

26. P. C. Ashok, B. B. Praveen, N. Bellini, A. Riches, K. Dholakia, and C. S. Herrington, "Multi-modal approach using Raman spectroscopy and optical coherence tomography for the discrimination of colonic adenocarcinoma from normal colon," Biomedical Optics Express 4, 2179-2186 (2013).

27. C. A. Patil, N. Bosschaart, M. D. Keller, T. G. van Leeuwen, and A. Mahadevan-Jansen, "Combined Raman spectroscopy and optical coherence tomography device for tissue characterization," Optics Letters 33, 11351137 (2008).

28. C. A. Patil, J. Kalkman, D. J. Faber, J. S. Nyman, T. G. van Leeuwen, and A. Mahadevan-Jansen, "Integrated system for combined Raman spectroscopy-spectral domain optical coherence tomography," Journal of Biomedical Optics 16, 011007 (2011).

29. C. A. Patil, H. Kirshnamoorthi, D. L. Ellis, T. G. van Leeuwen, and A. Mahadevan-Jansen, "A clinical instrument for combined raman spectroscopy-optical coherence tomography of skin cancers," Lasers in Surgery and Medicine 43, 143-151 (2011). 
30. S. Narendran, Q. Ji, D. Y. Eric, J. L. Alexander, C. L. Dina, E. P. Raphael, et al., "Line-scan Raman microscopy complements optical coherence tomography for tumor boundary detection," Laser Physics Letters 11,105602 (2014).

31. J. A. Izatt, and M. A. Choma, "Theory of Optical Coherence Tomography," in Optical Coherence Tomography, W. Drexler, and J. Fujimoto (Eds.), Springer, Berlin Heidelberg, 47-72 (2008).

32. T. Bocklitz, A. Walter, K. Hartmann, P. Rösch, and J. Popp, "How to pre-process Raman spectra for reliable and stable models?" Analytica Chimica Acta 704, 47-56 (2011).

33. C. G. Ryan, E. Clayton, W. L. Griffin, S. H. Sie, and D. R. Cousens, "SNIP, a statistics-sensitive background treatment for the quantitative analysis of PIXE spectra in geoscience applications," Nuclear Instruments and Methods in Physics Research Section B: Beam Interactions with Materials and Atoms 34, 396-402 (1988).

34. J. Zhao, H. Lui, D. I. McLean, and H. Zeng, "Real Time Raman Spectroscopy for Noninvasive in vivo Skin Analysis and Diagnosis," in Recent Advances in Biomedical Engineering, In-tech, Vienna, Austria, 455-474 (2010).

\section{Introduction}

Cancer is one of the leading causes of death [1]. The specific type and grade of tumors are of vital interest to determine the appropriate medical treatment for cancer patients. The term benign is given to tumors when they do not have the tendency to spread whereas cancerous refers to those that may spread and develop tumors in other parts of the body (metastasis). This grading system, which is essential for further treatment depends mainly on the location, size and growth of the tumor as well as on the extent of cancer invasion into the lymph system (nodes) or on metastasis. Based on the tumor grading further treatments such as surgery, chemo- or radiation therapy will be evaluated [2]. Since grading is crucial, well qualified histopathologists conduct assessments which are subjective and can have a large interoperator variation [3]. Endoscopy has not been satisfactory for an accurate disease detection [4]. Currently existing imaging techniques such as widefield detection, often coupled with fluorescence and staining agents, have low specificity and/or sensitivity. Confocal microscopy on the other hand allows cellular information but it is time consuming and requires training. Elastic scattering spectroscopy is under development but it requires many spectral acquisitions and might have a low accuracy to precede clinical trials $[5]$.

New non-invasive imaging strategies are required for an intraoperative tumor grading i.e. for differentiation between tissue types and providing valuable visual information especially about the depth of tumor penetration and dimensions. Within the last years optical coherence tomography (OCT) has shown its potential for optical guidance during surgeries [6]. For example, OCT is able to identify non-melanoma skin cancers and pre-cancers in vivo [7, 8]. In the case of basal cell carcinoma OCT images were successfully correlated with histopathological data [9-12]. Apart from OCT providing morphological information [13] spectroscopic approaches such as fluorescence imaging $[14,15]$, fluorescence lifetime imaging [16] and Raman spectroscopy [17] allow for retrieving molecular tissue information and therefore offer the potential for tumor grading [18]. Especially Raman spectroscopy has been proven as a powerful spectral histopathological tool for taking optical biopsies without the need for extraction of samples from patients $[19,20]$. Thus, tumor grading and identification of tumor types [21-23] can be applied based on the acquired Raman data. For a large variety of different cancer types, such a Raman-based spectral histopathology has been demonstrated in literature [24, 25]. However, the major drawback of Raman spectroscopy is its low sensitivity resulting in acquisition times of several seconds per spectrum hindering imaging large areas. Therefore, the combination of a highly specific technique such as Raman spectroscopy with a fast "red flag technique" such as OCT is desirable. This combination is able to locate tissue borders or suspicious regions based on OCT imaging which are subsequently chemically analyzed by Raman spectroscopy. Such a combination features the scanning speed of OCT imaging and the specificity of Raman spectroscopy. Previous work on the combination of Raman spectroscopy and OCT using chemometry has proven to be promising and shows higher classification accuracies although the measurements were carried out on separate instruments [26].

The combination of OCT and Raman spectroscopy was pioneered by the group Anita Mahadevan-Jansen $[27,28,29]$. Within their research, different wavelengths for both modalities were chosen i.e. 785 $\mathrm{nm}$ excitation for Raman spectroscopy and $855 \mathrm{~nm}$ central wavelength for OCT which enabled the use of a single detector for both spectral regions [28]. In general a high penetration and imaging depth is desirable for detecting tumor margins since deeper structures need to be resolved as seen in skin cancer [29, 30]. Therefore, the OCT/RS approach presented in the following utilizes a spectrometer based system with $1325 \mathrm{~nm}$ central excitation wavelength for OCT. The OCT system achieves an imaging depth of $7 \mathrm{~mm}$ in air and $2.5 \mathrm{~mm}$ in tissue limited by the radiation penetration depth. The presented OCT/RS setup has been implemented with commercially available parts only. Several imaging objective lenses were characterized with respect to their feasibility in terms of throughput 

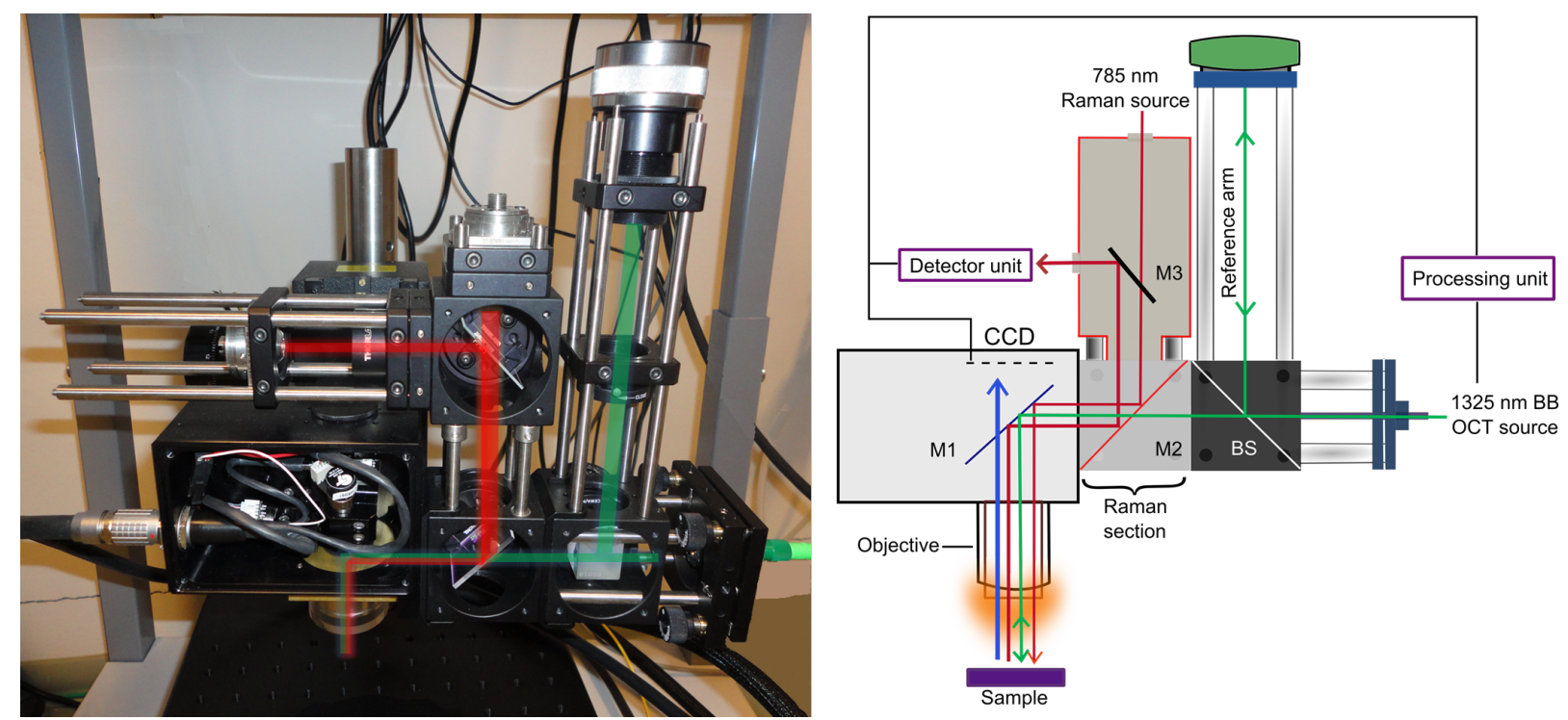

Fig. 1 Photograph of the OCT/RS system, where the different beam paths are visualized (A) and sketch of the combined OCT/RS system (B). M1, M2 and M3 represent the mirrors and BS denotes the beam splitter cube.

and background signals for both modalities. To demonstrate the feasibility of the OCT/RS setup for skin measurements different pork skin samples were analyzed.

\section{Method}

\subsection{Raman spectrometer system}

A Raman spectrometer (RAMAN RXN1, Kaiser Optical systems, USA) equipped with a $785 \mathrm{~nm}$ multimode laser source (Invictus ${ }^{\mathrm{TM}} 785-\mathrm{nm}$ NIR laser, Kaiser Optical Systems, Inc.,) was used. Measurements with the tested objective lenses (see section 3.2) resulted in an output laser power of $\sim 40 \mathrm{~mW}$ at the sample plane which was kept constant for all experiments. The integration time was 1 second during all experiments. The Stokes scattered Raman light was dispersed by a HoloPlex, holographic transmission volume grating and imaged on a CCD camera (Andor iDus 420 BB-DR).

\subsection{OCT system}

The spectral domain (SD) OCT device from Thorlabs (TelestoII $1325 \mathrm{LR}$ ) is equipped with a $1325 \mathrm{~nm}$ broadband superluminescent diode (SLD), with a bandwidth of $200 \mathrm{~nm}$. The excitation wavelength of $1325 \mathrm{~nm}$ allows for deep tissue penetration due to low scattering effects [31]. The reference arm, objective, CCD for recording visual images and the scanning optics are all integrated into a probe head, which will be explained in detail in the next section. The ThorImage OCT software was used to view and to adjust the image contrast of $3 \mathrm{D}$ surface images post acquisition. The device is capable of taking A- (single point), B- (line) and C- (3D volumetric) scans.

\subsection{OCT probe head}

The OCT probe head consists of a galvanic scanning unit, an objective or an achromatic doublet lens (see section 3.2) surrounded by an LED illumination ring for better visualization of the sample and a reference arm. The galvanic scanning unit consists of two scanning mirrors (M1 in Fig. 1B) for beam positioning in the $x-y$ sample plane. The 'slow' scanning mirror was modified that it transmits visible light received from the sample onto a CCD chip in order to be able to record visual images. The second 'fast' galvanic mirror was used to scan the other coordinate of the $x-y$ sample plane. The OCT source and spectrometer are coupled to the probe head by a FC/APC fiber. The light from the super luminescent diode (SLD) is collimated and directed to a beam splitter cube (BS in Fig. 1B) where the beam is split 50/50 into the sample and reference beam. The sample beam is directed to the galvanic scanning unit and then passes the objective. The back reflected sample light is collected through the objective, merged with the light from the reference arm and recoupled into the FC/APC fiber towards the OCT spectrometer. The optimum OCT reference intensity is regulated with the variable aperture placed in the reference arm.

\subsection{OCT/Raman device implementation}

In order to combine both OCT and Raman spectroscopy we used a probe cage consisting of the OCT components from Thorlabs (see sections 2.2 and 2.3) which was modified to incorporate the Raman modality (see Fig. 1A).

We have separated the OCT and Raman beam paths using dichroic mirrors at certain junctions where both beam paths cross (see Fig. 1B): 
- M 1 (AHF Analysentechnik, Tübingen, Germany) represents two galvo scanning mirrors one of which is a dichroic mirror allowing visible light $(\sim 350-650 \mathrm{~nm}$, blue arrow, Fig. 1B) to pass to the inspection CCD while reflecting $700-1500 \mathrm{~nm}$ with reflectivities higher than $93 \%$ (see Fig. 1B).

- M 2 (AHF Analysentechnik, Tübingen, Germany) redirects the Raman excitation and collection (750 $1050 \mathrm{~nm}$ ) onto the OCT beam path while remaining the OCT path unchanged. (Fig. 1B)

- M 3 (AHF Analysentechnik, Tübingen, Germany) separates the back reflected Raman signal from the Raman laser source. (Fig. 1B)

The alignment procedure proceeded with first aligning the OCT beam path such that the OCT laser beam was collimated onto the optical axis of the beam splitter cube and the reference arm (green beam path in Fig. 1A). Then mirror 2 was inserted and aligned such that the Raman beam path overlaps with the OCT beam path (overlaid beam path in Fig. 1A). Finally mirror 3 was aligned using a neon calibration lamp to achieve highest collection efficiencies (red beam path, in Fig. 1A). The reference arm length was adjusted according to the objective lens used (see section 3.2).

\section{Experimental Section}

\subsection{Sample preparation}

For a first validation of both subsystems (OCT and Raman) commercially available pork loin of $\sim 1 \mathrm{~cm}$ thickness consisting of bone, fat and muscle tissue regions was used as a test sample. The pork loin sample was placed on a petri dish and characterized by the combined Raman - OCT (OCT/RS) system. For each measurement a freshly sectioned sample was used. A customized Labview program was used to scan a $10 \mathrm{x}$ $10 \mathrm{~mm}$ pork loin sample to obtain a 3D OCT surface image by acquiring B-scans at sagittal sections. Subsequently the selected sample area was scanned with RS using $100 \times 100$ pixels with an integration time of 1 second per pixel. These measurements allow for a comparison between different objective lenses (see sections 3.2 and 4.1). The depth resolution achieved for the tested objective lenses (see section 3.2 and 4.1) was compared by analyzing B-scans of scotch tape. Finally pork skin samples with visual defects were used for realistically simulating skin measurements. These samples were extracted from pork belly, which was made for consumption.

\subsection{Objective lenses}

The two modalities OCT and Raman have contrary optical demands. While deep tissue OCT imaging requires long distance objectives with a rather low numerical aperture, Raman-spectroscopy on the other hand relies on high numerical apertures to achieve high collection efficiencies. The perfect objective for combining OCT and Raman measurements is therefore always a tradeoff between both modalities. However, for both cases an anti-reflection coating transmitting wavelengths from $700-1500 \mathrm{~nm}$ is advantageous. Initially we tested the following three different commercially available microscope objectives specially designed for OCT imaging systems and optimized for IR-OCT imaging:

- LSM02: Thorlabs, $f=18 \mathrm{~mm}, \mathrm{NA}=0.11$, magnification of 10x, working distance $7.5 \mathrm{~mm}$

- LSM03: Thorlabs, $f=36.0 \mathrm{~mm}, \mathrm{NA}=0.056$, magnification of $5 \mathrm{x}$, working distance $25.1 \mathrm{~mm}$

- LSM04: Thorlabs, $f=54.0 \mathrm{~mm}, \mathrm{NA}=0.037$, magnification of $3 \mathrm{x}$, working distance $42.0 \mathrm{~mm}$.

Additionally an achromatic doublet lens (35mmNIRII; Edmund optics, US) with AR coating was tested, all with respect to achieve optimum Raman intensities, low Raman backgrounds as well as clear OCT images (see section 4.1). The three objectives are telecentric, which ensures minimal image distortions and a constant image resolution due to the spot size being constant in the flat image plane while scanning. Each time the objective was changed, the reference arm length was adjusted accordingly. In addition the probe head was calibrated for each objective prior to the measurement.

\subsection{Data analysis}

The analysis of the Raman spectra was carried out according to a standard protocol recently developed in our group [32]. First a wavenumber calibration was carried out based on Paracetamol as wavenumber standard. Thereafter, a baseline subtraction with the SNIP algorithm [33] was applied and a vectornormalization was performed. As analysis procedure, a $\mathrm{k}$-means cluster analysis with $k=5$ was applied in order to highlight spectral changes within the dataset. This kmeans cluster analysis was carried out to allow for a comparison between the white light image, OCT scan and the Raman data. In general the generation of the false-color Raman images is not necessary and single point measurements are sufficient. Here Raman images were only generated to verify that with the measurement system, OCT and Raman images can be recorded for the same field-of-view. In future clinical applications, Raman spectra will be collected pointwise from regions of interest identified by a large OCT image.

\section{Results and discussion}

\subsection{Objective lens comparison}

As mentioned in section 3.2. OCT has contrary demands as compared to Raman spectroscopy with respect to the applied objective lenses. For Raman spectroscopy the applied objectives should show a good collection efficiency as well as a low Raman background signal, enabling cluster algorithms to differentiate different tissue types. To quantify the applicability of all objective lenses introduced in section 3.2 pork loin samples consisting of bone, muscle and fat tissue were measured and analyzed with both modalities. Fig. 2 shows OCT and Raman measurements recorded with 
LSM03- 3x

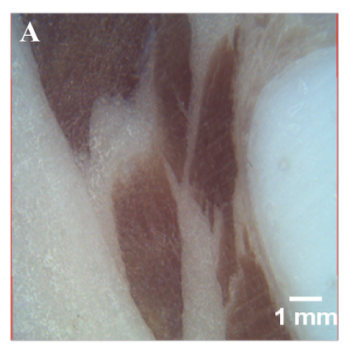

LSM04- 5x

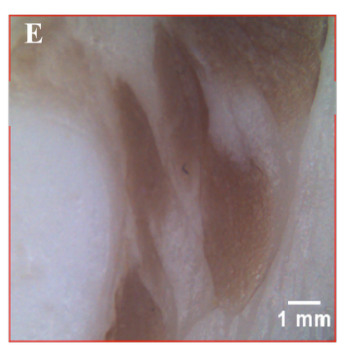

Achromatic doublet lens

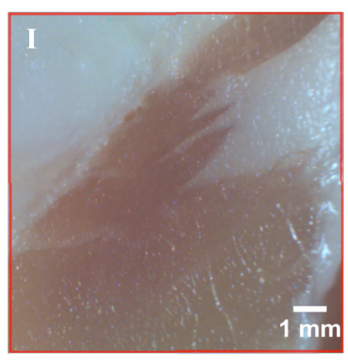

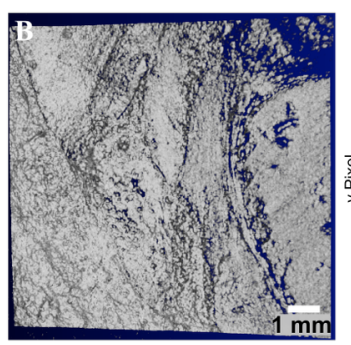
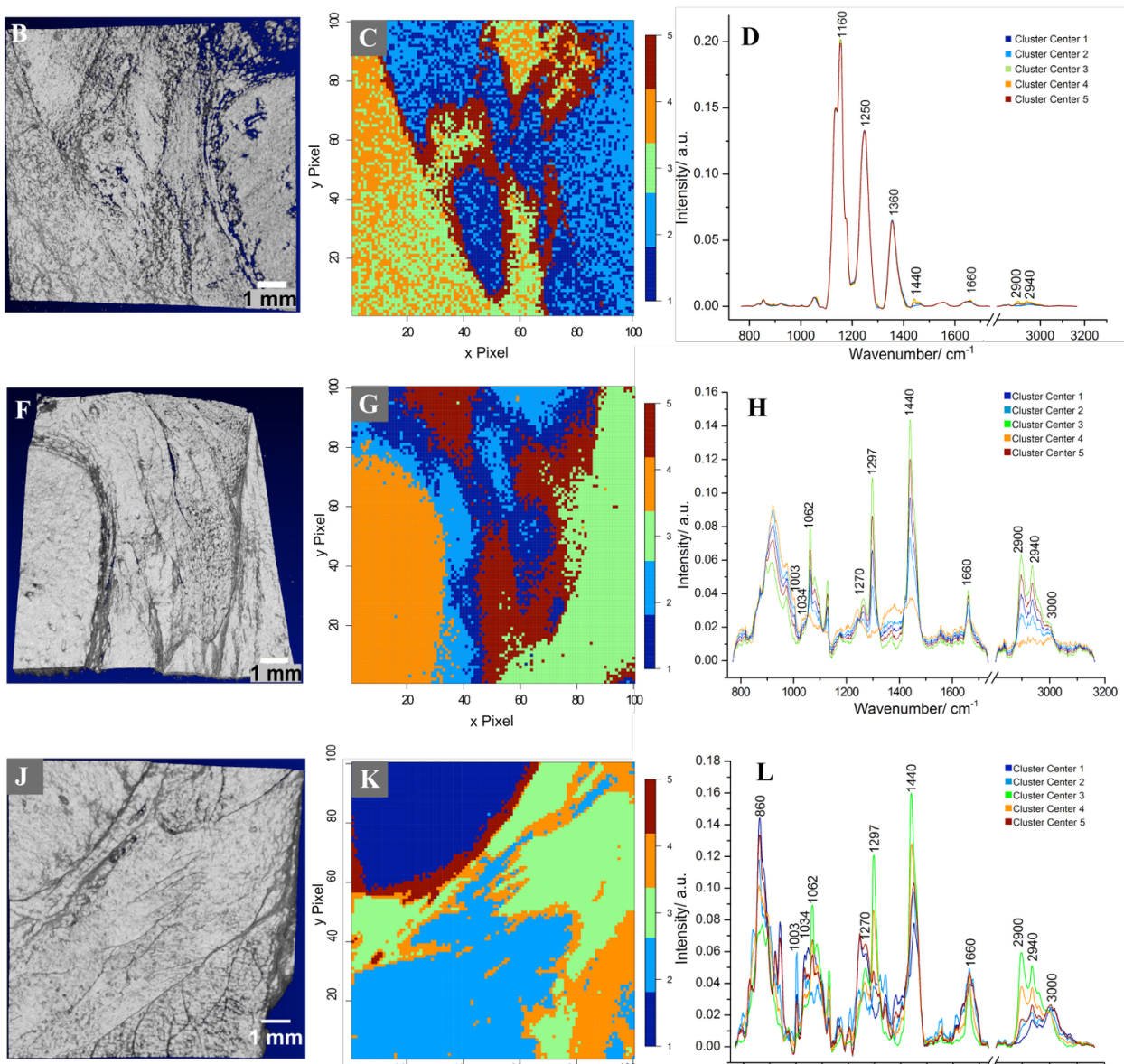

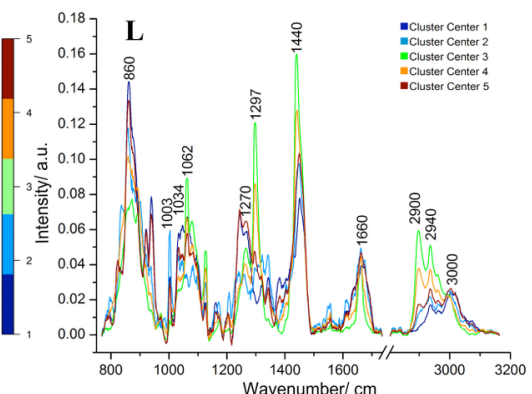

Fig. 2 Evaluation of different objective lenses (see section 3.2) for combined OCT/Raman measurements using pork loin samples. The rows are representing the results for each individual objective lens. The first column shows the microscopic white light images of the sample area (A, E, I). The second column shows the OCT C-scan as seen from the surface (B, F, J). The third row shows the k-means cluster image derived from the Raman raster scan of the same area $(C, G, K)$ and the right row shows the corresponding cluster mean Raman spectra (D, H, L).

the two objectives (LSM03 and LSM04) and the achromatic doublet lens described in section 3.2. The objective LSM02 exhibiting the highest numerical aperture of all commercially available OCT objectives and therefore representing the best tradeoff for both modalities, had to be excluded from the study since it only transmits $10 \%$ of the $785 \mathrm{~nm}$ Raman excitation laser. Fig. 2 shows white light images acquired with the probe head (left column), the corresponding top view CScans from the same area (second column), the resulting $\mathrm{k}$-means Raman cluster images from raster scans of that area (third column) and the corresponding mean Raman spectra for each cluster (right column) for the two objectives LSM03, LSM04 and the achromatic doublet lens.

The white light images recorded with all three objective lenses highlight the sample distribution of fat, bone and muscle tissue. It is worth mentioning here, that the used galvanic mirror reflecting all wavelength higher than $650 \mathrm{~nm}$ has an impact on the sample image quality in a way that the red channel intensity is reduced, which will be also important for the experiments reported in section 4.3. The OCT C-Scans contain the complete volumetric $3 \mathrm{D}$ information of the tissue but are here displayed as 2D top view images for a better comparison with the morphology displayed in the white-light images. Both modalities (OCT and white light) show a good agreement with respect to the sample morphology.

The Raman spectra recorded with LSM03 show large Raman background signals of the objective itself that disturb the Raman spectra of the sample i.e. an overlap with the Raman bands that are particularly important for the differentiation between different tissue types. Here the clusters 3 and 4 displayed in Fig. 2 C seem to be distributed throughout the regions consisting of fat, while the clusters 1 and 2 can be mainly found within the muscle and bone regions. Cluster 5 depicts the meat- fat boundary. The three very intense Raman bands at 1160,1250 and $1360 \mathrm{~cm}^{-1}$ observed when using the LSM03 objective (Fig. 2D) are most probably due to organic compounds present in the optical glue of the objective itself. Moreover, the loss of laser power due to the objectives is quite significant and only $30 \%$ 

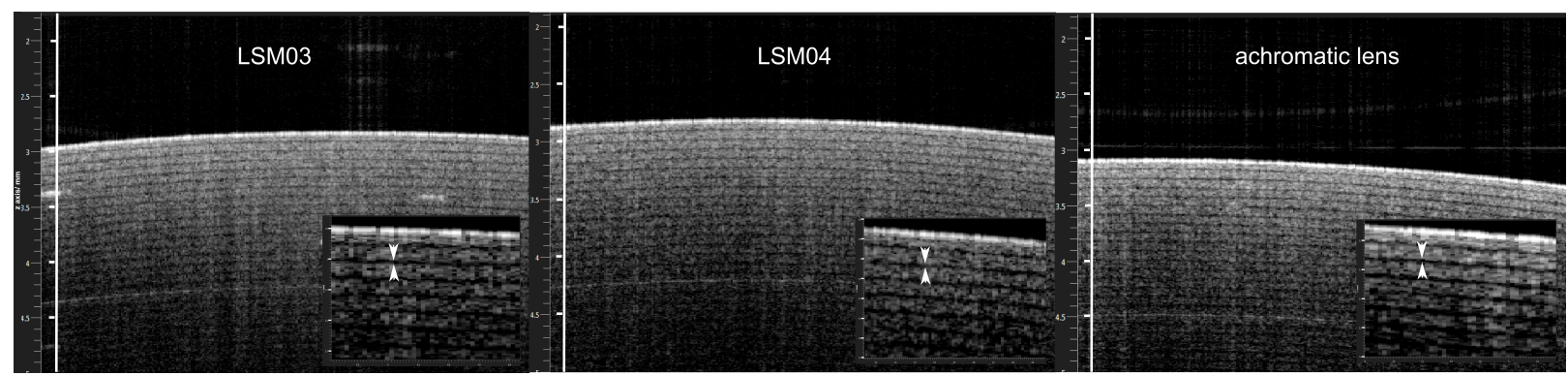

Fig. 3 Comparison of the axial resolution using scotch tape as a test sample with LSM03, LSM04 and the achromatic lens doublet as objective lenses. Inset: the adhesive in between tape layers was measured to be $\sim 10 \mu \mathrm{m}$ shown by the white arrows.
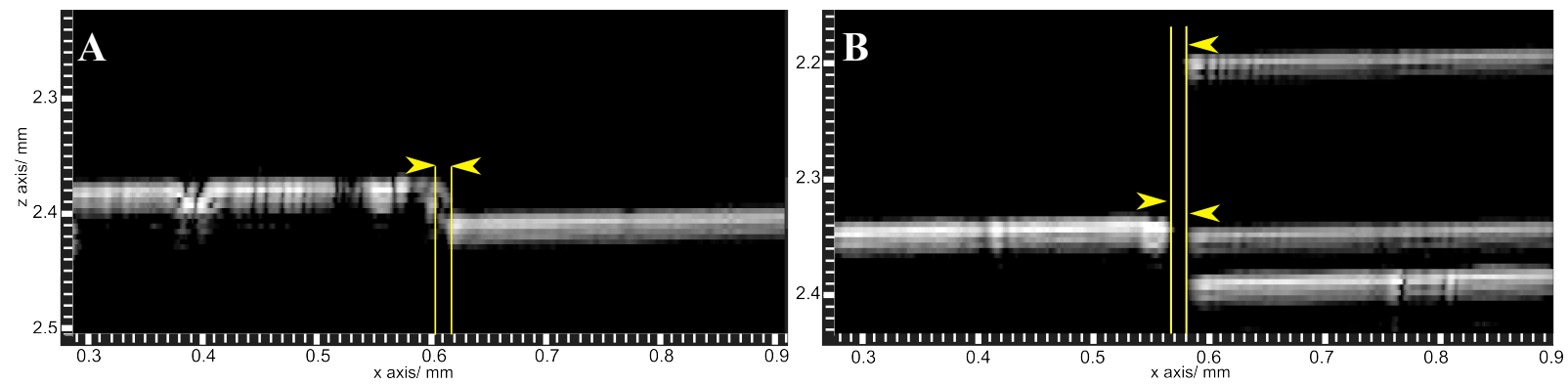

Fig. 4 B-scans of tape on a glass slide (A) and glass cover slip on a glass slide (B) to determine the lateral resolution of OCT in combination with the achromatic doublet. The mismatch of the proximal and distal surfaces shown by the yellow arrows was found to be $13 \mu \mathrm{m}$ in both cases.

of the Raman excitation power is transmitted to the sample. These results clearly show that the objective LSM03 is not suitable for combined OCT/Raman measurements.

The microscope objective LSM04 shows only minimal background Raman signatures (see Fig. 2H) due to the objective itself and therefore leads to more significant Raman images (see Fig. 2G) as compared to LSM03. The application of LSM04 allows for clustering bone, fat and muscle tissues. The k-means Raman cluster image clearly correlates with the white-light and the OCT image (Figs. 2 E-G). Fig. 2G shows that cluster 1 relates to muscle tissue. The transition region between fat and muscle is separated by cluster 2 displayed in cyan. Bulk fat is highlighted by cluster 3 in green and bone is denoted by the $4^{\text {th }}$ cluster plotted in orange. However, the overall transmission of the Raman excitation laser for this objective LSM04 is only $25 \%$. This low transmission in combination with the rather low numerical aperture leads to a signal to noise ratio of 64.3 (calculated for the Raman peak at $1440 \mathrm{~cm}^{.1}$, Fig. 2 $\mathrm{H})$.

The achromatic doublet lens has a transmission loss of only $10 \%$ of the Raman excitation laser and therefore a much higher transmission for the whole Stokes Raman scattered light between 785 to $1050 \mathrm{~nm}$ as compared to the two microscope objectives LSM03 and LSM04. Therefore the achromatic doublet lens shows a good Raman collection efficiency while exhibiting the same numerical aperture than LSM03. The k-means cluster image (Fig. 2K) recorded with the achromatic doublet lens shows that all the pork tissue components can be better differentiated than for the LSM objectives. This is directly linked to the higher signal to noise ratio of the acquired Raman spectra. One drawback when using the doublet lens was that it prevented the ThorImage software from conducting its image calibration accurately. However, due to its superior Raman performance while simultaneously maintaining the quality of OCT scans, this achromatic doublet lens represents a very suitable tradeoff for both subsystems i.e. Raman and OCT. Overall, the SNR achieved with the doublet lens $(\mathrm{SNR}=248.5)$ is 4 time better than the SNR obtained with the LSM04 $(\mathrm{SNR}=64.3)$.

The axial resolution of the OCT subsystem is only dependent on the source bandwidth and the OCTspectrometer resolution. These two properties do not depend on the applied objectives as can be seen in Fig. 3 where we analyzed B-scans of scotch tape.

Figure 3 shows that all three objective lenses exhibit a comparable depth resolution. Therefore, the achromatic lens doublet was chosen as the best suited objective lens for combining OCT and Raman. The following system characterization measurements were performed with the achromatic doublet lens only.

The lateral resolution of the OCT part in combination with the achromatic doublet lens was characterized by B-scans over a $50 \mu \mathrm{m}$ tape, which was placed on a glass slide with the assumption that the tape has a straight edge. The resulting mismatch between the proximal and distal surfaces of the tape was found to be about $13 \mu \mathrm{m}$ (Fig. 4 A).

In order to confirm this lateral resolution determined for the tape, a glass cover slide was also placed on a 

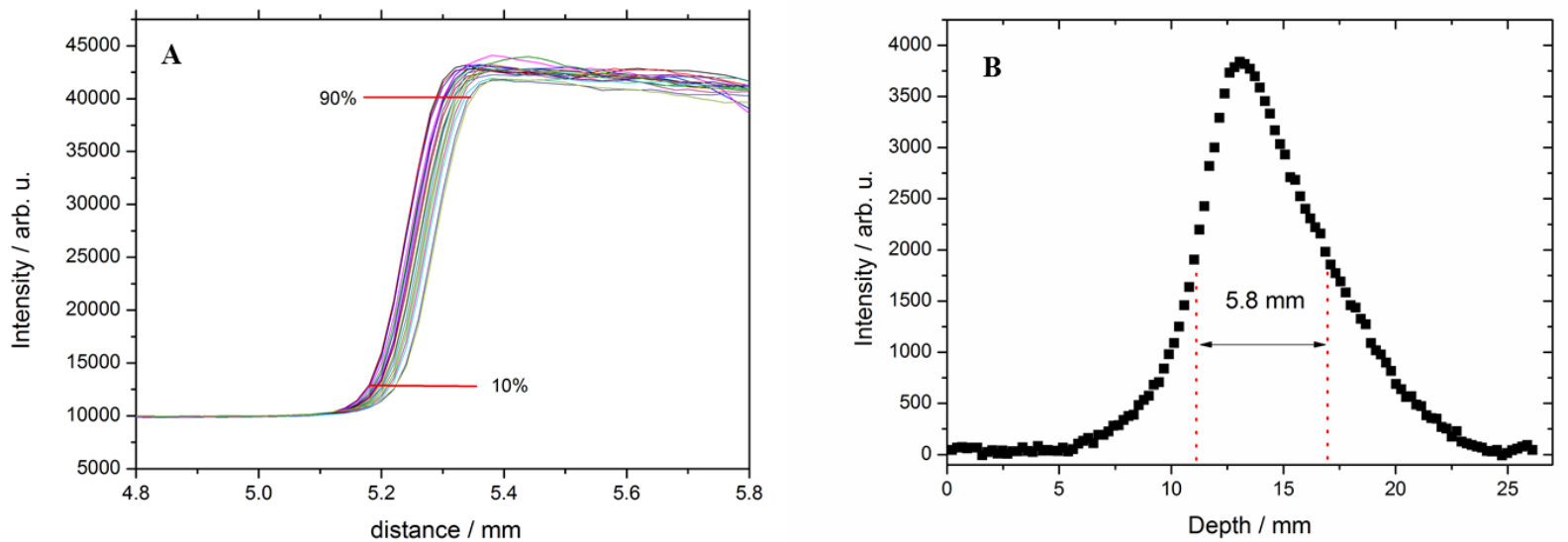

Fig. 5 Lateral resolution measured between 10 and $90 \%$ of the integrated Teflon Raman peak at $338 \mathrm{~cm}^{-1}$ (A) of a Teflon layer and depth of field measurement (B) measured for a Raman peak at $493 \mathrm{~cm}^{-1}$ for scotch tape.

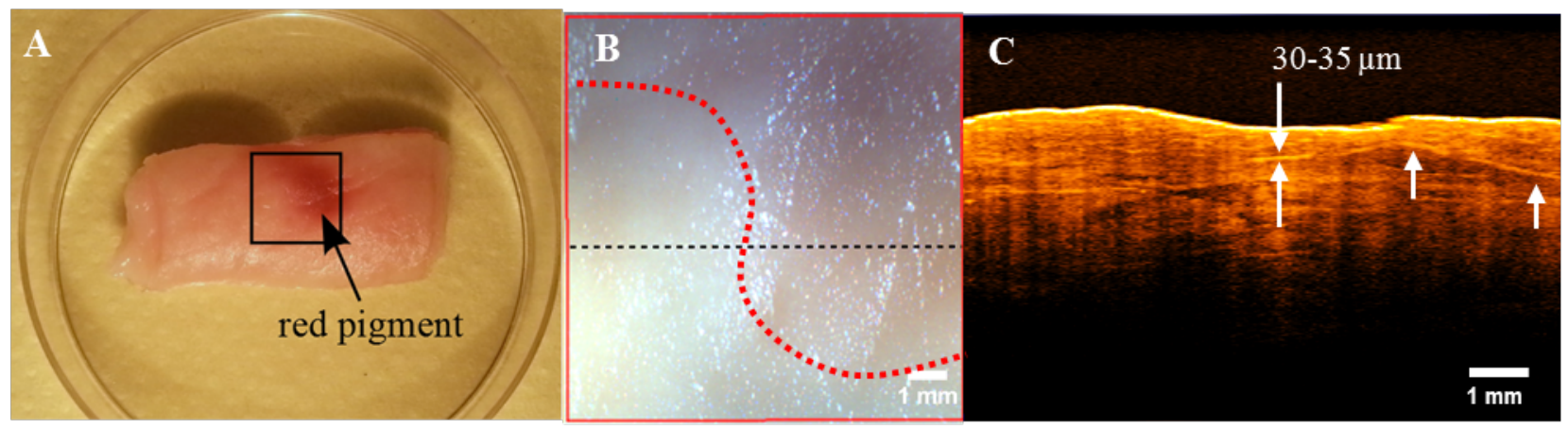

Fig. 6 Pork skin with a red pigment (A), zoom into a 10x10 mm region of interest (B) and a B-scan of the dashed red line shown in $\mathrm{B}$ emphasizing the position of the red pigment within the skin tissue $(\mathrm{C})$.

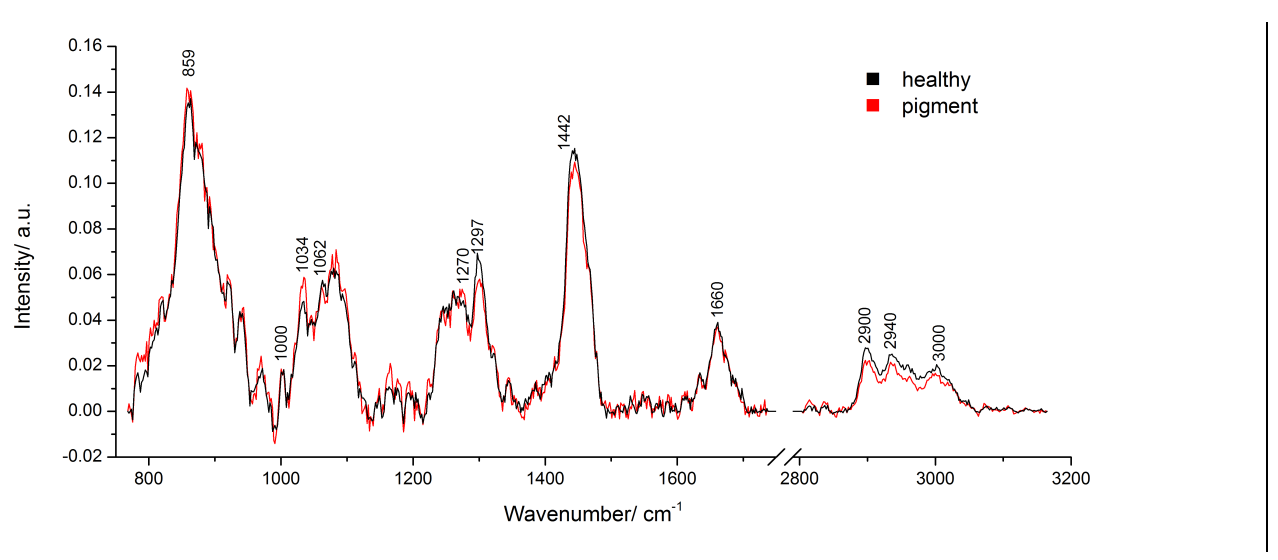

Fig. 7 Mean Raman spectra differentiating healthy and red pigmented areas on the pork skin sample shown in Fig. 6. Prominent Raman peaks are labeled (for details see text).

microscopy glass slide (Fig. 4B) and the mismatch between the proximal and distal surfaces was determined in analogy to the tape (Fig. 4A). This analysis also reveals a lateral resolution of $13 \mu \mathrm{m}$.

\subsection{Raman characterization}

In order to characterize the lateral resolution of the Raman modality with the achromatic lens doublet a raster scan was performed on a thin Teflon layer. The step size of the scan was set to $20 \mu \mathrm{m}$ and a map with a dimension of $20 \times 500$ pixels was collected. Subsequently the generated image was analyzed for intensity variations at the representative Teflon Raman peak at $338 \mathrm{~cm}^{-1}$. The lateral resolution was defined as the intensity variation from $10 \%$ to $90 \%$ of the integrated peak area, as shown in Fig. 5A resulting in an achievable resolution of $104 \pm 6 \mu \mathrm{m}$ for the Raman part, 
which is in agreement to the core diameter of the Raman excitation fiber of $105 \mu \mathrm{m}$.

To characterize the depth of focus a thin layer $(100$ $\mu \mathrm{m})$ of transparent scotch tape was measured over a length of $27 \mathrm{~mm}$ on the z-axis. Several Raman spectra in several focal positions were measured with a distance of $0.225 \mathrm{~mm}$ between consecutive points. Subsequently the data set was analyzed for intensity variations of the scotch tape Raman peak at $493 \mathrm{~cm}^{-1}$ and the depth of focus (DOF) was set to the full width half maximum of the peak intensity as depicted in Fig. $5 \mathrm{~B}$ resulting in a DOF of $5.8 \mathrm{~mm}$. In conclusion these studies resulted in a sample penetration depth limited DOF of about $1 \mathrm{~mm}$ for $785 \mathrm{~nm}$ [34]. The focal volume thus can be visualized as having a high resolution laterally $(\sim 100$ $\mu \mathrm{m})$ and a low axial resolution $(\sim 700-1000 \mu \mathrm{m})$.

\subsection{Skin tissue measurements}

For validating our combined OCT/RS setup for later skin and skin cancer applications a pork skin sample with a red pigment on the surface was chosen as a model for skin studies. Fig. 6 displays the white-light images (Figs. 6A and B) as well as a B-scan through the sample area (Fig. 6C). The axial resolution of the OCT system was $9 \mu \mathrm{m}$ allowing for the observation of morphological details of layer variations beneath the pork skin as can be seen in Fig. 6C.

It is obvious that the wavelength cutoff of the used galvanic mirror, which reflects all wavelengths higher than $650 \mathrm{~nm}$, has an impact on the sample image quality and the pigmented area is barely visible in the whitelight image shown in Fig. 6B. The tissue boundary is visualized by the red dotted line and the OCT B-Scan course shown in Fig. 6C is indicated by the black dotted line. The B-scan displayed in Fig. 6C shows a single tissue layer highlighted with a white arrow. Therefore we conclude that the red structures are a subcutaneous hemorrhage of the skin. The defect has a layer thickness of about $30-40 \mu \mathrm{m}$. Therefore ten Raman spectra inside the pigmented area as well as outside with a margin of $1 \mathrm{~mm}$ were recorded in order to analyze this red pigmented area in more detail on a molecular level. The mean Raman spectra derived from the ten spectra which were acquired from the hemorrhage and the skin region with a margin of $1 \mathrm{~mm}$ are shown in Fig. 7.

The main difference, that can be seen within the Raman spectra is a loss in intensity of $\mathrm{CH}$-related modes $\left(1298,1443,2850-3000 \mathrm{~cm}^{-1}\right)$. Skin mainly consists of fat. Due to the narrowness of the hemorrhage $(35 \mu \mathrm{m})$ in comparison to the DOF $(\sim 700-800 \mu \mathrm{m})$, no obvious spectral components of the Heme-groups are visible. All expected Raman spectral features of skin (Amide I (1660), Amide III (1270), Nucleic Acids (1030) and Phenylalanine (1000)) are clearly visible and in agreement with the literature [28, 29].

\section{Conclusion}

The manuscript reports the successful implementation of a combined OCT/Raman device that is able to perform OCT scans and Raman maps or single point Raman measurements on bulk tissue samples. The most suitable optical system is an achromatic lens doublet, which represents a compromise between high OCT source penetration in tissue and high Raman signal collection efficiency. Furthermore this lens has a minimal intrinsic Raman background and showed a larger signal to noise level for the Raman part as compared with common OCT objectives. The 3D scans obtained from the OCT system can also provide axial (depth) information with a resolution of $\sim 10 \mu \mathrm{m}$. By adjusting the image contrast post-acquisition, it is possible to clearly view the margins between different tissue types that would essentially help the user to better identify any abnormalities and the penetration depth of these. Essentially, this device is able to carry out fast scans to gather morphological information with OCT that helps the user identify abnormalities and then take pointwise Raman spectra for molecular diagnostics of the concerned region at a slower rate. One drawback of the system is the low axial resolution of the Raman part which is penetration depth limited to $700-1000 \mu \mathrm{m}$ depending on the tissue type. This will have an impact on the model classification accuracies (e.g. cancer vs. non-cancer) since not only the tissue layer of interest can be measured. Hence, the Raman spectra are the result of a superposition of the Raman information of the different tissue layers. On the other hand, the system allows acquiring Raman spectra with short integration times and high SNRs from any point of large sample areas.

The optical setup can be potentially employed to characterize the surface of pathological tissue such as diseased skin tissue or to monitor wound healing processes. Having the advantage of being able to view cross sectional images via OCT and then carrying out diagnostic measurements with Raman spectroscopy would aid the user in making accurate diagnostic decisions. Further studies must be conducted to investigate the feasibility as well as the specificity and sensitivity of such a system.

\section{Acknowledgments}

Financial support by the Carl- Zeiss Stiftung is greatly acknowledged. 